\title{
The Mathematics of Joseph Bernstein
}

\section{Roman Bezrukavnikov $^{1}$. Alexander Braverman ${ }^{2}$. Michael Finkelberg ${ }^{3}$ • Dennis Gaitsgory ${ }^{4}$}

Published online: 4 November 2016

(C) Springer International Publishing 2016

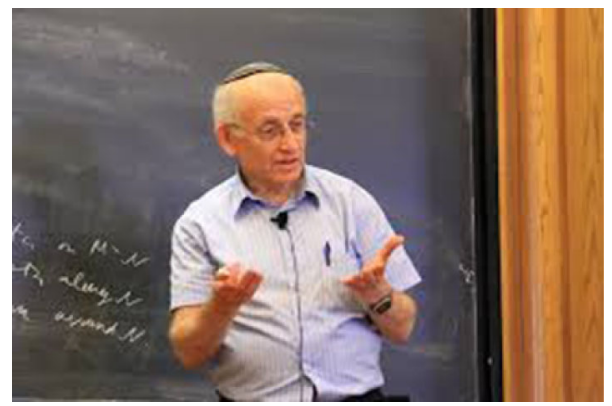

Joseph Bernstein's lecture at a recent conference

Joseph Bernstein is one of those few mathematicians whose contributions have changed the landscape of mathematics, primarily in the fields of representation theory

Roman Bezrukavnikov

bezrukav@math.mit.edu

Alexander Braverman

sashabraverman@gmail.com

Michael Finkelberg

fnklberg@gmail.com

Dennis Gaitsgory

gaitsgde@math.harvard.edu

1 Department of Mathematics, MIT, 77 Massachusetts Avenue, Cambridge, MA 02139, USA

2 Department of Mathematics, University of Toronto, Perimeter Institute for Theoretical Physics and Mathematics, Bahen Centre, 40 George Street, Toronto, ON M5S 2E4, Canada

3 Department of Mathematics, National Research University, Higher School of Economics, 6 Usacheva Street, Moscow 119048, Russian Federation

4 Department of Mathematics, Harvard University, One Oxford Street, Cambridge, MA 02138, USA 
and algebraic geometry, contributing to an interface of these two fields in what is known today as geometric representation theory.

Joseph's first breakthrough happened in the early seventies when he invented the theory of algebraic D-modules. The initial impetus for the development of the theory was the desire to answer a question posed by I. M. Gelfand about analytic continuation of a certain generalized function depending on a parameter. That function was a solution of a particular differential equation and Joseph's idea was to capture the relevant features of this differential equation by a certain algebraic structure, and that structure turned out to be that of a module over the ring of differential operators. Algebraic D-modules turned out to be a fundamental mathematical notion, and subsequent developments took it into many different directions, beyond being a tool for the study of differential equations. This theory was also independently discovered by the Kyoto school of M. Sato, M. Kashiwara and T. Kawai.

One can study the category of all D-modules on a given scheme, and the interaction between such categories under morphisms of schemes. This point of view makes D-modules into a sheaf theory. Moreover, this sheaf theory is related to another fundamental sheaf theory of a topological nature - that of constructible sheaves. This link between the algebro-analytic and the topological worlds, called the RiemannHilbert correspondence, was established by Joseph Bernstein (and, independently, by M. Kashiwara, P. Schapira and F. Mebkhout).

Transferring the abelian category of D-modules to the constructible side, A. Beilinson, J. Bernstein, P. Deligne and O. Gabber discovered the notions of a $t$-structure on a triangulated category and of a perverse sheaf, which provided an algebraic formalism for the intersection cohomology developed by M. Goresky and R. MacPherson; shortly thereafter these notions quickly spread throughout mathematics.

In a different direction, D-modules on varieties acted upon by an algebraic group (e.g., the flag variety) are closely related to modules over the Lie algebra of that group. In the specific case of the flag variety, this relationship can be made into an equivalence of categories: this is the localization theorem of A. Beilinson and J. Bernstein.

A combination of the above ideas: perverse sheaves, Riemann-Hilbert correspondence and localization led to the proof (by Joseph Bernstein in collaboration with A. Beilinson, and independently by J.-L. Brylinski and M. Kashiwara) of the KazhdanLusztig conjecture.

In the 1970s, Joseph Bernstein in collaboration with I. M. Gelfand and S. Gelfand, worked on the theory of highest weight representations of a semisimple Lie algebra. Their series of papers was arguably the most substantial development in our understanding of the algebraic structure of representations, perhaps since the discovery of the structure of finite-dimensional representations in the 1920s-1930s.

No less significantly, the papers of Bernstein, Gelfand and Gelfand uncovered the pattern of highest weight categories, which forms the core of an important chapter in representation theory today, as many of the representation categories that we know (quantum groups, modular representations, etc.) belong to that class.

In parallel with the 1970s and 1980s, Joseph made a series of groundbreaking discoveries in the representation theory of p-adic groups. This started from his proof of the Type-I property of the representations of a reductive group. Characteristically, Bernstein's proof of an impressive specific result was followed by his reworking foun- 
dations of the theory into a beautiful and conceptually clear picture. He explained that the entire category of representations splits into cuspidal components, and that each cuspidal component is governed by an associative algebra that is a finite and flat module over its center (=the Bernstein center of the category); the Type-1 property mentioned above manifests itself in that, for a fixed congruence level, only finitely many of these components survive.

In the process, Joseph systematized the study of induction and Jacquet functors (the "geometric lemma"), which allowed him (jointly with Zelevinsky) to reduce the classification of irreducible representations of $G L_{n}$ to cuspidal ones and create the tools for studying this question for a general reductive group. He also discovered the unexpected fundamental "second adjointness" (in the case of admissible representations discovered independently by W. Casselman) and proved the trace Paley-Wiener theorem, and also greatly clarified the situation with the Plancherel theorem.

Recently, in a series of works with A. Reznikov, Joseph introduced new representation theoretic methods into the analytic theory of automorphic forms. A notable application of these methods is a proof of subconvexity bounds for central values of some automorphic L-functions, improving the well-known Phragmén-Lindelöf convexity bound: finding such bounds is one of the central problems of analytic number theory.

Joseph has also obtained important results in the theory of supermanifolds and Lie super-algebras (partly joint with D. Leites) and algebraic geometry, such as in his paper with I. M. Gelfand and S. Gelfand on vector bundles on a projective space.

Joseph's contribution to mathematics goes well beyond his papers; his unique mentoring talents and generosity in sharing ideas led to his profound influence over numerous mathematicians of several generations.

P. Erdos introduced a famous image of Proofs from the Book, proofs that feel as if they inherently belonged to the statement, exposing its nature. While many proofs by Bernstein undoubtedly belong to that category, what's more remarkable is that whole theories pioneered by Bernstein, or rather his very approach to mathematics is the "approach from the Book". It is guided by the vision of mathematics, however difficult it may be, as governed by ultimately simple and elegant principles that are there for the mathematician to discover. It is this vision that inspired and will continue to inspire generations of mathematicians. 